\title{
Determination of Antioxidant Properties and Antimicrobial Activity of Vinyl Phenolic Compounds Extracted from Saccharomyces cerevisiae Against Uropathogenic Bacteria
}

\section{MUAZZAM SHERIFF MAQBUL ${ }^{1}$, AEJAZ A. KHAN² ${ }^{2}$ TASNEEM MOHAMMED ${ }^{2}$, S. M. SHAKEEL IQUBAL ${ }^{2 *}$, IBRAHIM AHMED SHAIKH ${ }^{3}$, UDAY M. MUDDAPUR ${ }^{4}$, GOUSE BASHA SHEIK $^{5}$, S. K. SINGH ${ }^{6}$, MOHAMMED SHAHID HUSSAIN ${ }^{7}$ and MOHAMMED GAMAL ${ }^{8,9}$}

${ }^{1}$ Department of Microbiology and Immunology, Ibn Sina National College for Medical Studies, Jeddah, Kingdom of Saudi Arabia.

${ }^{2}$ Department of General Science, Ibn Sina National College for Medical Studies, Jeddah, Kingdom of Saudi Arabia.

${ }^{3}$ Department of Pharmacology, College of Pharmacy, Najran University, Najran, Saudi Arabia. ${ }^{4}$ Department of Biotechnology, KLE Technological University, BVB Campus, Hubballi, 580031, India. ${ }^{5}$ Department of Microbiology, College of Applied Medical Sciences, Ad-Dawadmi, Shaqra University, Saudi Arabia.

${ }^{6}$ Department of Chemistry, GGV (Central University), Bilaspur (C.G) - 495009, ndia. ${ }^{7}$ Department of Orthodontics and Dentofacial Orthopedics, M. A. Rangoonwala Dental College, Pune, Maharashtra, India.

${ }^{8}$ Pharmaceutical Chemistry Department, Pharmacy college, Jouf University, P.O. Box 2014, Sakaka, Aljouf, Kingdom of Saudi Arabia.

${ }^{9}$ Pharmaceutical Analytical Chemistry Department, Faculty of Pharmacy, Beni-Suef University, Alshaheed Shehata Ahmed Hegazy St., 62574, Beni-Suef, Egypt.

${ }^{\star}$ Corresponding author E-mail: shakeeliqubal @ gmail.com

http://dx.doi.org/10.13005/ojc/360104

(Received: November 25, 2019; Accepted: January 05, 2020)

\section{ABSRTACT}

The aim of research study of determining the vinyl phenolic compounds with antioxidant properties and antimicrobial activity using acetone and methanol extracts of Saccharomyces cerevisiae (S. cerevisiae). The HPLC-UV technique was employed for the identification of the vinyl phenolic compounds and Ferric and Ferrous reducing anti-oxidant power assay along with radical scavenging methods were applied to determine the anti-oxidant properties of the yeast extract. The biochemical tests showed the presence of alkaloids, reducing sugars, steroid, proteins, phenol, cardiac glycosides. Further antimicrobial properties of the yeast extract using Kirby-Bauer disc diffusion method indicates, E.coli exhibits the best susceptibility towards the yeast extract. The antimicrobial susceptibility was excellent for all the isolated uropathogens when compared with the standard antibiotics. The metabolites produced by the yeast exhibits vital pharmaceutical important substances such as analgesic, antipyretic, anti-proliferative and antimicrobial properties. This study is a small attempt towards a larger future to serve the mankind with natural remedies.

Keywords: Vinyl Phenolic compound, Antioxidant Properties, Antimicrobial Activity, Saccharomyces cerevisiae, Uropathogenic Bacteria.

This is an Open Access article licensed under a Creative Commons license: Attribution 4.0 International (CC- BY). Published by Oriental Scientific Publishing Company @ 2018 


\section{INTRODUCTION}

The major objective of this work is to determine the vinyl phenolic compounds with antioxidant properties of this yeast by acetone and methanol extracts of $S$. cerevisiae and determine the antimicrobial activity $y^{1,2,3,4}$. The HPLC-UV technique was employed for the identification of the vinyl phenolic compounds present in the yeast extract ${ }^{5,6,7,8}$. The Ferric and Ferrous reducing anti-oxidant power assay along with radical scavenging method using 1,1-diphenyl-2-picryl hydrazyl were applied to determine the anti-oxidant properties of the yeast extract $^{4,9,10,12}$. The biochemical properties of the yeast extract were determined by different chemical methodologies ${ }^{13,14,15}$. The antimicrobial properties of the yeast extract was determined by the standard antibiotic sensitivity test methodology designed by the Kirby and Bauer ${ }^{7,8,16}$. The reactive oxygen species generally gets produced by the cellular metabolism of aerobic bacteria in which the molecular oxygen plays a vital role ${ }^{11,17}$. They are contributors for the signal transduction of various cellular physiological processes for the human. The diseases such as cardiovascular, Alzheimer, Parkinson, Rheumatoid arthritis, etc. can be caused due to the excess production of reactive oxygen species beyond the threshold antioxidant capacity limit of our biological system which can lead to oxidative stress ${ }^{14,18}$. The oxidative stress can be minimized by restoring the balance between the oxidant and antioxidant in the human system for the proper function of the physiological system ${ }^{14}$. The administration of synthetic antioxidant substances can be a remedy but also results in harmful side effects which can affect the human organs due to its residual effect. In the recent studies the harmful effect of the synthetic chemical compounds on humans was once again debated and hence there is a need for the alternate solution ${ }^{7,16}$. The alternative solutions should be able to restore the balance between the oxidant and antioxidant in the human system for the proper function of the physiological system and at the same time it should not cause any negative harmful side effects like that of the synthetic compounds ${ }^{14}$. The best solution to overcome is to rediscover the nature blessed natural resources used by the ancestors to diagnose various dangerous diseases ${ }^{7,8}$. The natural products should also be considered only if it is as effective as the synthetic chemical substances ${ }^{9}$. The commonly available brewer's yeast used in the brewing industries proved to be one of the best natural substance to overcome the synthetic chemical substances and is much more effective ${ }^{3,4,9}$. This study is intended to study this yeast's potential reactive oxygen capacity. The brewer's yeast employed to our study is $S$. cerevisiae. This yeast possess vinyl phenol compounds 4-vinylphenol and 4-vinylguaiacol in $1: 1$ ratio which promotes it to be one of the best biopharmaceutical substance $e^{4,11,13}$. The enzymatic activity of $S$. cerevisiae is excellent with substituted cinnamatecarboxy-lyase which converts coumaric and ferulic acids into vinylphenols by its capability of transforming non-oxidative decarboxylation of phenolic acid ${ }^{18,19,20}$. This endo-cellular activity of this yeast is a potential source which can be used for the prophylaxis of oxidative stress related disorders ${ }^{14}$. The metabolites produced by the yeast exhibits vital pharmaceutical important substances such as analgesic, antipyretic, anti-proliferative and cytotoxic antimicrobial properties ${ }^{7,8}$. The antimicrobial property of the yeast extract was experimented against uropathogenic bacterial infections from isolated specimens collected, purified and identified from the urinary tract infected patients $\mathbf{s}^{7,8,21}$.

\section{MATERIALS AND METHODS}

\section{Reagents}

Chemicals like methanol, DPPH, acetone, $\mathrm{FeCl}_{3}$, Ninhydrin reagent, Fehlings, Benedicts, Salkowski's reagents were procured from SigmaAldrich chemicals (USA). Microbiological selective isolation culture media along with biochemical identification reagents and standard antimicrobial discs were employed. All other chemicals were analytical grade.

\section{Yeast collection and Identification}

A purified and isolated standard strain sample of the yeast $S$. cerevisiae from one of the ATCC (American Type Collection Centre) specimen collection centre in India and was used for this experiment. The confirmation identification for the pure culture of the yeast was verified by employing various in vitro fungal identification technique.

\section{Preparation of the yeast extracts}

$10 \mathrm{~g}$ of the dried yeast samples were sequentially extracted with $250 \mathrm{~mL}$ of acetone and methanol at $37^{\circ} \mathrm{C}$ for 24 hours ${ }^{1,2}$. A rotatory evaporator was employed to dry the concentrated 
extract under reduced pressure with the temperature of $40^{\circ} \mathrm{C}$ and stored in the sterile test tubes at $-20^{\circ} \mathrm{C}$. The yield of respective yeast extracts were estimated as Yield $(\%)=($ dry weight of extract/dry weight of samples) $\times 100$

\section{Identification of the vinyl phenolic compound in the yeast}

Based on Huneck and Yoshimura the identification of the vinyl phenolic compounds present in the yeast $S$. cerevisiae were performed by HPLC-UV technique analytical technique ${ }^{20}$ The stored dried yeast extracts were dissolved in $500 \mu \mathrm{L}$ of acetone and analyzed by using Agilant Technologies, 1200 Series HPLC instrument with C18 column $25 \mathrm{~cm} \times 4.6 \mathrm{~mm}, 10 \mu \mathrm{m}$ with UV spectrophotometric detector.

\section{Determination of anti-oxidant properties in the yeast}

1) Based on the Oyaizu Ferric and Ferrous Reducing-Antioxidant Power (FRAP) assay methodology the anti-oxidant properties of yeast extract of $S$. cerevisiae was determined by the reducing powers of the dried yeast extracts by using various concentrations of the extract ranging from 50 to $1000 \mu \mathrm{g} /$ $\mathrm{mL}$ diluted with saline buffer $0.2 \mathrm{M}, \mathrm{pH} 6.6$ \& $2.5 \mathrm{~mL}$ of ferric chloride (1\%) and ferrous solution (1\%) respectively and incubated at $50^{\circ} \mathrm{C}$ for 30 minute. After incubation period $2.5 \mathrm{~mL}$ of $10 \%$ trichloroacetic acid (TCA) was added to the mixture to stop the reaction. The mixture was centrifugated at $3000 \mathrm{~g}$ for $10 \mathrm{~min}$ and $2.5 \mathrm{~mL}$ distilled water added to 25 $\mathrm{mL}$ of the supernatant with $0.5 \mathrm{~mL} \mathrm{FeCl}_{3}(0.1 \%)$ and then the optical density was observed at $700 \mathrm{~nm}$ with an American made UNICO spectrophotometer which determined that the higher absorbance of the reaction mixture which indicates greater reducing power of the yeast. Suitable positive and negative controls were used for the test. The test was repeated for the five times and the mean value was recorded for the accuracy of the results.

2) Based on Kosanic et al., radical scavenging methodology using 1,1-diphenyl-2-picryl hydrazyl, the anti-oxidant properties of the yeast extract of $S$. cerevisiae to scavenge DPPH free radicals was estimated by the reduction of the reaction color between
DPPH solution and sample extracts was determined by adding $2 \mathrm{~mL}$ of $0.12 \mathrm{mM}$ solution DPPH in methanol to the $1 \mathrm{~mL}$ of various concentrations ranging from 50 - 1000 $\mu \mathrm{g} / \mathrm{mL}$ of the yeast extract respectively. The mixture was incubated at $37^{\circ} \mathrm{C}$ for $30 \mathrm{~min}$ and the absorbance of the reaction mixture was measured at $517 \mathrm{~nm}$ with an American made UNICO spectrophotometer to determine the radical scavenging activity of the yeast extract. Suitable positive and negative controls were used for the test. The test was repeated for the five times and the mean value was recorded for the accuracy of the results.

\section{Bio-chemical analysis of the yeast extract}

The biochemical properties of the yeast extract of $S$. cerevisiae was determined by Mayer's test, Fehling's test, Salkowski's test and Ninhydrin test, Ferric Chloride test, Libermann Burchard's Test, Benedict's Test, Keller-kilani test and ammonia test ${ }^{13}$.

\section{Antimicrobial Property of the yeast extract}

The antimicrobial activity of the yeast extract was performed against the clinically isolated uropathogens from the urinary tract infected persons.

\section{Isolation and Identification of uropathogenic Specimen}

The clinical urinary sample was collected from the suspected urinary tract infected patients and inoculated on the selective media Cystine Lactose Electrolyte Deficient Agar with $\mathrm{pH}$ indicator bromothymol blue. The inoculated plates were incubated at $37^{\circ} \mathrm{C}$ for $24 \mathrm{~h}$ and biochemical reaction test were done for all the isolated uropathogens from the isolated colonies by using IMVIC test and results were observed respectively ${ }^{21}$.

\section{Antibiotic Susceptibility testing by Kirby-Bauer Disc Diffusion Method}

Based on the standard antibiotic sensitivity methodology developed by Kirby and Bauer the antimicrobial activity of the yeast extract were determined ${ }^{7,8,16}$ by incorporating the standard antibiotic discs coated with the yeast extract on the various Mueller Hinton agar plates inoculated with different uropathogenic samples such as E.coli, Klebsiella Sp., Pseudomonas Sp. and Proteus $S p$. isolated from the clinical urine specimens respectively. The inoculated plates impregnated 
with the antibiotic disc of yeast extract were incubated for $24 \mathrm{~h}$ at $37^{\circ} \mathrm{C}$. The zone formation around the disc indicates the susceptibility of the organism and no zone formation indicates the resistance of the organism towards the antibiotic disc of the yeast extract.

\section{RESULTS AND DISCUSSION}

The yeast extract results were exemplary for all the tests performed. Based on Huneck and Yoshimura the identification of the vinyl phenolic compounds present in the $S$. cerevisiae were performed by the HPLC-UV analytical technique analysis of acetone and methanol extracts of the yeast extract was used to identify their major vinyl phenolic compounds Figure 1.

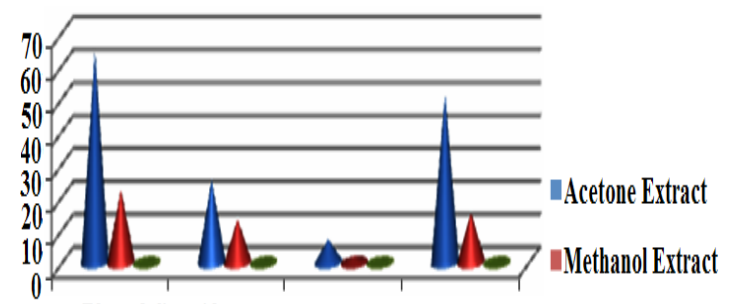

Physodalic acid Atranorin Chloratranorin Control Rilffer

Fig.1. Analysis of a vinyl phenolic compounds present in the Saccharomyces cerevisiae (acetone and methanol extracts) by HPLC-UV technique

By comparing the retention times (tR) yeast extract were identified. The main compounds in acetone extract of yeast were physodalic acid (tR $=3.295 \pm 0.012 \mathrm{~min})$, atranorin $(\mathrm{tR}=8.642 \pm 0.020$ $\mathrm{min}$ ) and chloratranorin ( $\mathrm{tR}=11.918 \pm 0.026 \mathrm{~min})$. The most abundant substance in the acetone yeast extract was physodalic acid where as in methanol yeast extract only small amount of physodalic acid was observed in the chromatogram. It is evidenced in the chromatograms of ferric and ferrous reducing power assay of oxidant/antioxidant based on Oyaizu technique as shown in Figure 2.

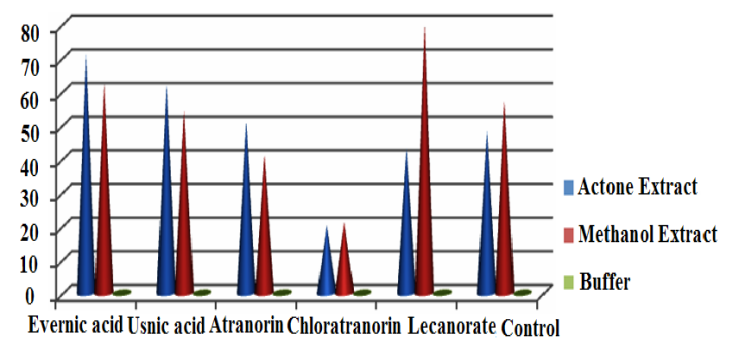

Fig. 2. Chromatograms of ferric and ferrous reducing power assay of oxidant/antioxidant
The chromatogram showed evernic acid, (tR $=3.045 \pm 0.029 \mathrm{~min})$, usnic acid (tR $=7.854$ $\pm 0.025 \mathrm{~min}$ ), atranorin ( $\mathrm{tR}=8.236 \pm 0.028 \mathrm{~min}$ ) and chloratranorin ( $\mathrm{tR}=11.554 \pm 0.028 \mathrm{~min}$ ) are present in the acetone extracts. The most abundant compound in acetone extract is evernic acid while in methanol extract also evernic acid and usnic acid detected but the methyl lecanorate (tR $=1.515 \pm$ $0.009 \mathrm{~min}$ ) is the most abundant compound.

Based on Kosanic' et al., radical scavenging methodology using 1,1-diphenyl-2-picryl hydrazyl, the anti-oxidant properties of the yeast extract of $S$. cerevisiae to scavenge DPPH free radicals was estimated by the reduction of the reaction color between DPPH solution and sample extracts was determined results showed a statistically significant data as the antioxidant activity was elevated from $2.01 \pm 0.009 \%$ to $86.14 \pm 0.013 \%$ in accordance with the increase of the concentration of the extracts from 50 to $1000 \mu \mathrm{g} / \mathrm{mL}$ of yeast extract Figure 3 .

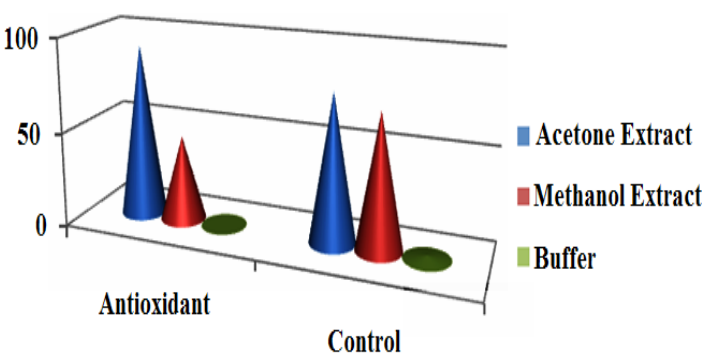

Fig. 3. Radical scavenging methodology using 1,1-diphenyl-2-picryl hydrazyl, the anti-oxidant properties of the yeast extract of Saccharomyces cerevisiae to scavenge DPPH free radicals

The acetone yeast extract with $1000 \mu \mathrm{g} /$ $\mathrm{mL}$ showed largest DPPH radical scavenging activity: $86.14 \pm 0.013 \%$. According to test results the antioxidant activities obtained from the methanol yeast extracts were obtained but lower than that measured for the acetone yeast extract which showed the highest DPPH radical scavenging activity with an $\mathrm{IC}_{50}=240.220 \pm 15.165 \mu \mathrm{g} / \mathrm{mL}$.

The biochemical properties analysis of the yeast $S$. cerevisiae extract showed the presence of alkaloids, reducing sugar, steroids, protein, phenol, glycosides, cardiac glycosides and amino acids as shown in Table 1. 
Table 1: Biochemical properties of yeast

\begin{tabular}{cccc}
\hline Test & Observation & Result & Interpretation \\
\hline Mayer's test & Formation of a creamy substance & Positive & Presence of alkaloids. \\
Fehling's test & Brick red color formation & Positive & Presence of reducing sugar \\
Salkowski's test & Reddish brown color formation & Positive & Presence of steroids \\
Ninhydrin test & Violet color formation & Positive & Presence of protein \\
Ferric chloride test & Blue black coloration & Positive & Presence of Phenol \\
Libermann Burchard's test & Violet to bluish green color formation & Positive & Presence of Glycosides \\
Benedict's test & Formation of orange red precipitate & Positive & Presence of reducing sugar \\
Keller-kilani test & Brown ring at the interface & Positive & Presence of Cardiac glycosides \\
Ammonia test & Yellowish color formation & Positive & Presence of Amino Acids \\
\hline
\end{tabular}

Further, collected specimens from the urinary tract infected persons were isolated by culturing on a selective media Cystine Lactose Electrolyte Deficient Agar with $\mathrm{pH}$ indicator Bromothymol blue showed Yellow lactose fermenting colonies for the Klebsiella $s p$. and E.coli whereas blue non lactose fermenting colonies for the Proteus sp. and Pseudomonas $s p$. The isolated organisms were confirmed using biochemical reactions of IMVIC and the result observed were tabulated (Table 2) and confirmed the identification of the pathogens.

Table 2: biochemical assessment of the isolated uropathogens

\begin{tabular}{|c|c|c|c|c|c|c|}
\hline Organism & Indole & Methyl red & Voges proskauer & Citrate & Urease & Oxidase \\
\hline \multirow[t]{2}{*}{ E.Coli } & pink ring & red color & yellow color & green color & yellow color & no purple color \\
\hline & Positive & positive & negative & negative & negative & negative \\
\hline \multirow[t]{2}{*}{ Klebsiella sp. } & yellow ring & yellow color & red color & blue color & pink color & no purple color \\
\hline & Negative & negative & positive & positive & positive & negative \\
\hline \multirow[t]{2}{*}{ Pseudomonas sp. } & yellow ring & yellow color & yellow color & blue color & yellow color & purple color \\
\hline & Negative & negative & negative & positive & negative & positive \\
\hline \multirow[t]{2}{*}{ Proteus sp. } & yellow ring & red color & yellow color & blue color & pink color & no purple color \\
\hline & Negative & positive & negative & positive & positive & negative \\
\hline
\end{tabular}

Then using the Kirby-Bauer disc diffusion method the antimicrobial susceptibility of the prepared yeast extract antibiotic disc were tested against the isolated uropathogens and the results were exemplary. E.colishown the best susceptibility towards the yeast extract while the Pseudomonas $s p$. show the least susceptibility. The Klebsiella sp. and Proteus sp. shown a moderate susceptibility with the yeast extract antibiotic disc. The antimicrobial susceptibility was excellent for all the isolated uropathogens when compared with the standard antibiotics (Table 3).

Table 3: Antimicrobial susceptibility comparison of yeast extract with standard antibiotics for urinary tract infections

\begin{tabular}{|c|c|c|c|c|}
\hline Antimbiotics & E.coli & Klebsiella sp. & Pseudomonas sp. & Proteus sp. \\
\hline Yeast Extract & Sensitive $25 \mathrm{~mm}$ & Sensitive $23 \mathrm{~mm}$ & Sensitive $19 \mathrm{~mm}$ & Sensitive $21 \mathrm{~mm}$ \\
\hline Trimethoprim & Sensitive $21 \mathrm{~mm}$ & Intermediate $15 \mathrm{~mm}$ & Resistant 2mm & Intermediate $14 \mathrm{~mm}$ \\
\hline Sulfamethoxazole & Sensitive $18 \mathrm{~mm}$ & Sensitive 24mm & Resistant 4mm & Sensitive 19mm \\
\hline Fosfomycin & Sensitive $21 \mathrm{~mm}$ & Sensitive $22 \mathrm{~mm}$ & Sensitive $21 \mathrm{~mm}$ & Sensitive $18 \mathrm{~mm}$ \\
\hline Nitrofurantoin & Sensitive $26 \mathrm{~mm}$ & Sensitive $21 \mathrm{~mm}$ & Sensitive $20 \mathrm{~mm}$ & Sensitive $18 \mathrm{~mm}$ \\
\hline Azithromycin & Sensitive $24 \mathrm{~mm}$ & Sensitive $19 \mathrm{~mm}$ & Sensitive $22 \mathrm{~mm}$ & Sensitive $22 \mathrm{~mm}$ \\
\hline Ceftriaxone & Sensitive $19 \mathrm{~mm}$ & Sensitive $24 \mathrm{~mm}$ & Sensitive $20 \mathrm{~mm}$ & Sensitive $24 \mathrm{~mm}$ \\
\hline Cephalexin & Sensitive $20 \mathrm{~mm}$ & Sensitive $21 \mathrm{~mm}$ & Sensitive $18 \mathrm{~mm}$ & Sensitive $21 \mathrm{~mm}$ \\
\hline Levofloxacin & Intermediate $16 \mathrm{~mm}$ & Resistant 8mm & Resistant 2mm & Resistant 6mm \\
\hline Ciprofloxacin & Sensitive 22mm & Sensitive $19 \mathrm{~mm}$ & Intermediate $14 \mathrm{~mm}$ & Intermediate $13 \mathrm{~mm}$ \\
\hline
\end{tabular}




\section{CONCLUSION}

The antioxidant properties along with the vinyl phenolic compounds of the yeast Saccharomyces cerevisiae shows that this solated yeast extract can be used as a potential anti synthetic substance for the treatment of many diseases not only just as an antibiotic but can also help to balance between the oxidant and antioxidant level in the body for the proper function of the physiological system in maintaining the proper body functions as it contains many essential biochemical nutrients required for our body to produce reactive oxygen species to eradicate many diseases. This study is a small attempt towards a larger future to serve the mankind with natural remedies.

\section{ACKNOWLEDGEMENT}

We would like to Acknowledge our family members for their constant support and encouragement.

\section{Conflicts of interest}

No conflict of interest.

\section{REFERENCES}

1. Kapoor SL., Kapoor LD. On the botany and distribution of 'pashanbheda'. Sachitra Ayurved., 1976, 28(12), 769-791.

2. Singh AP. A Short review on Didymocarpuspedicellata: The Lithontriptic Ethnomedicine, Ethnobotanical Leaflets., 2007, 11, 73-75.

3. Mojab F., Kamalinejad M., Naysanch G., Hamid R. Phytochemical screening of some species of Iranian plants. Iranian Journal of Pharmaceutical Research., 2003, 2(2),77-82.

4. Awika JM., Rooney LW., Wu X., Prior RL., Cisneros-Zevallos L. Screening methods to measure antioxidant activity of sorghum (Sorghum bicolor) and sorghum products. Journal of Agricultural and Food Chemistry., 2003, 51, 6657-6662.

5. Huneck S., Yoshimura I. Identification of Lichen Substances, 1st ed, Berlin, SpringerVerlag., 1996.

6. Oyaizu M. Studies of products browning reaction: antioxidative activity of products of browning reaction prepared from glucosamine. Jpn J Nutr., 1986, 44, 307.

7. Muazzam SM., AlHasel HMB., Majid DH., Momen TN., AlHazmi HAM., AlJeddani FMS., AIMalaki RTW., Khan AA., Iqubal SMS. Chemical Analysis (GC-FID-MS) and Antimicrobial Activity of Parmotrema perlatum Essential Oil Against Clinical Specimens. Orient.J.Chem., 2019, 35(6), in press.

8. Gouse BS., Muazzam SM, Gokul SS, Ranjith MS. Isolation and characterization of actinomycetes from soil of ad-dawadmi,
Saudi Arabia and screening their antibacterial activities. Int $J$ of Pharm and Pharmaceutical Sc., 2017, 9(10),267-279.

9. Huang D., Ou B., Prior RL. The chemistry behind antioxidant capacity assays. Journal of Agricultural and Food Chemistry., 2005, 53, 1841-1856.

10. Ben Salah M., Abderraba M., Tarhouni MR., Abdelmelek $\mathrm{H}$. Effects of ultraviolet radiation on the kinetics of in vitro percutaneous absorption of lavender oil. Int J Pharm., 2009, 382, 33-38.

11. Poopal AC., Laxman RS. Studies on the biological reduction of chromate by Streptomyces griseous. J Haz Mat., 2009, 169, 539-545.

12. Adams RP. Identification of Essential Oil Compo- nents by Gas Chromatography/ Mass Spectroscopy, $4^{\text {th }}$ edition. Allured Publishing Corporation, Carol Stream, IL., 2007.

13. Kokate CK., Purohit AP., Gokhale SB. Pharmacognosy., $20^{\text {th }}$ Edn, Nirali prakashan, Pune., 2002, 108-109.

14. Kaur G., Lone IA., Athar M. Protective effect of Didymocarpuspdeicellata on ferric nitrilotriacetate (FeNTA) induced renal oxidative stress and hyperproliferative response, Chemico biological interaction., 2007, 165(1), 33-44.

15. Rathore JS., Garg SK., Gupta SR. A chalcone and flavones from Didymocarpuspedicellata. Phytochemistry., 1981, 20, 1755-1756. 
16. Bisht CMS., Iqubal SMS., Khan AA., Tasneem M., Dawoud A., Gamal M., Singh SK., Asghar $\mathrm{BH}$. Natural Products in Drug Discovery: Antibacterial and Antifungal Activity of Essential Oil of Compound Isolated from Senecio royleanus. J Pure Appl Microbio., 2019, 13(3), 1611-1617.

17. Arpin C., Quentin C., Grobost F., Cambau E., Robert J., Dubois V., Coulange L., Andre C. Nationwide survey of extendedspectrum \{beta\} lactamase producing Enterobacteriaceae in the French community setting. J Antimicrob Chemother., 2009, 63, 1205-1214.

18. Zhu M., Cui Y. Determination of 4-vinylgaiacol and 4-vinylphenol in top-fermented wheat beers by isocratic high performance liquid chromatography with ultraviolet detector. Braz arch biol technol., 2013, 56(6), 1018-1023.

19. Dey TB., Kuhad RC. Enhanced production and extraction of phenolic compounds from wheat by solid-state fermentation with Rhizopus oryzae RCK2012. Biotechnol Rep., 2014, 4, 120-127.

20. Bagewadi ZK., Muddapur UM., Madiwal SS., Mulla SI .,Khan AA. Biochemical and enzyme inhibitory attributes of methanolic leaf extract of Datura inoxia Mill. Environmental Sustainability., 2019, 2, 75-87.

21. Bahl CP., Seshadri TR. Pashanbhedi: drugs for urinary calculus, Eds., K.N. Udupa., 1978 7 7-98. 